\title{
Evaluation of Artery Visualizations for Heart Disease Diagnosis
}

\author{
Michelle A. Borkin, Student Member, IEEE, Krzysztof Z. Gajos, Amanda Peters, Dimitrios Mitsouras, \\ Simone Melchionna, Frank J. Rybicki, Charles L. Feldman, \& Hanspeter Pfister, Senior Member, IEEE
}

A
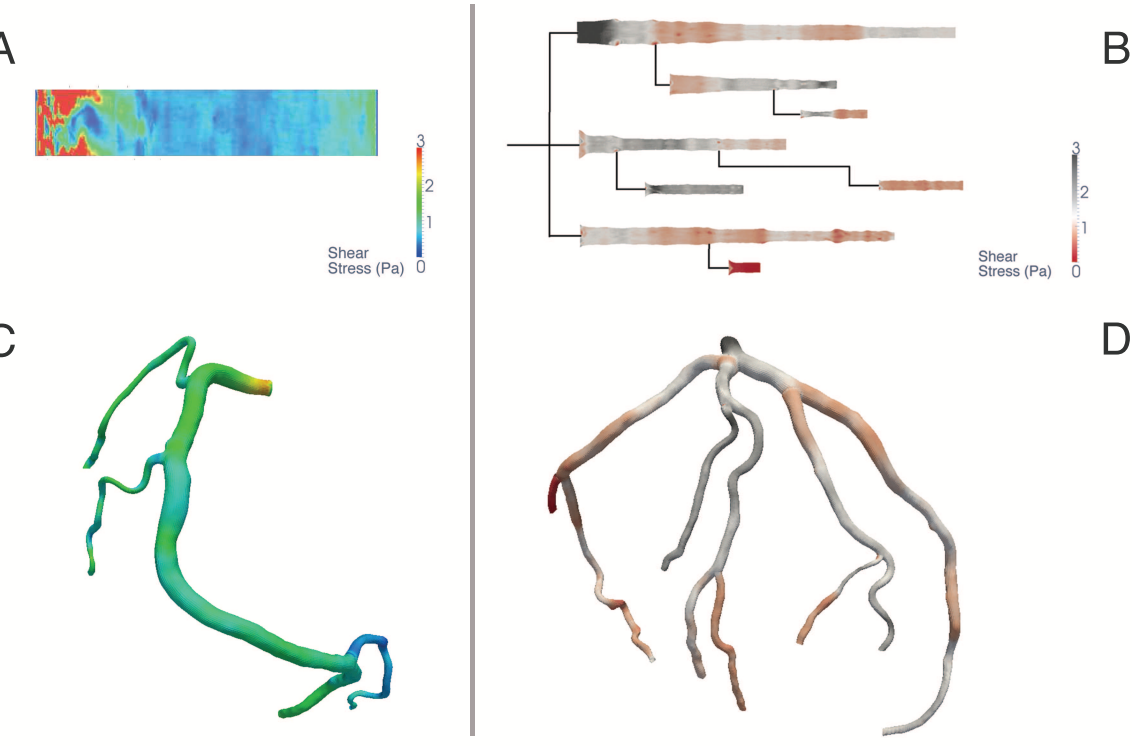

Fig. 1. Left: Traditional 2D projection $(A)$ of a single artery, and 3D representation $(C)$ of a right coronary artery tree with a rainbow color map. Right: 2D tree diagram representation (B) and equivalent 3D representation (D) of a left coronary artery tree with a diverging color map.

\begin{abstract}
Heart disease is the number one killer in the United States, and finding indicators of the disease at an early stage is critical for treatment and prevention. In this paper we evaluate visualization techniques that enable the diagnosis of coronary artery disease. A key physical quantity of medical interest is endothelial shear stress (ESS). Low ESS has been associated with sites of lesion formation and rapid progression of disease in the coronary arteries. Having effective visualizations of a patient's ESS data is vital for the quick and thorough non-invasive evaluation by a cardiologist. We present a task taxonomy for hemodynamics based on a formative user study with domain experts. Based on the results of this study we developed HemoVis, an interactive visualization application for heart disease diagnosis that uses a novel 2D tree diagram representation of coronary artery trees. We present the results of a formal quantitative user study with domain experts that evaluates the effect of 2D versus 3D artery representations and of color maps on identifying regions of low ESS. We show statistically significant results demonstrating that our 2D visualizations are more accurate and efficient than 3D representations, and that a perceptually appropriate color map leads to fewer diagnostic mistakes than a rainbow color map.
\end{abstract}

Index Terms-Quantitative evaluation, qualitative evaluation, biomedical and medical visualization.

\section{INTRODUCTION}

In the United States, the leading cause of death is heart disease resulting in over 600,000 deaths per year [24]. Early prevention and treatment is vital for saving lives, and visualization plays an essential role

- M. Borkin, K. Gajos, A. Peters, and H. Pfister are with the School of Engineering \& Applied Sciences, Harvard University, E-mail: \{borkin,kgajos,apeters,pfister\}@seas.harvard.edu .

- D. Mitsouras \& F. Rybicki are with the Applied Imaging Science Lab and C. Feldman is with the Vascular Profiling Lab at Brigham and Women's Hospital \& Harvard Medical School, E-mail: \{dmitsouras,frybicki,cfeldman\}@partners.org .

- S. Melchionna is with the IPCF-CNR, Consiglio Nazionale delle Ricerche, E-mail: simone.melchionna@romal.infn.it .

Manuscript received 31 March 2011; accepted 1 August 2011; posted online 23 October 2011; mailed on 14 October 2011.

For information on obtaining reprints of this article, please send email to:tvcg@computer.org. for patient diagnosis in cardiovascular imaging. A new non-invasive diagnostic technique under development uses Computed Tomography Angiography (CTA) data from patients combined with blood flow simulations to calculate hemodynamic risk factors, in particular Endothelial Shear Stress (ESS), in coronary arteries [40]. Visualization methods of this data are of great value for this emerging research and have the potential to lead to faster, more accurate heart disease diagnoses.

Current visualizations techniques, as shown in Fig. 1 (left), use either a $2 \mathrm{D}$ cylindrical projection of a single artery or a $3 \mathrm{D}$ representations of the coronary artery tree. In both cases, ESS is mapped to the surface using a color encoding, typically with a rainbow (or "spectrum") color map. Both representations have their advantages and disadvantages: 2D allows one to see all the data at once, but anatomical information is lost both in the shape of a vessel and in how each individual vessel connects to other branches. 3D preserves the anatomical structure, but introduces issues of occlusion and requires human interaction to rotate the model in order to see all the data. The fundamental visualization issue is how to display a scalar quantity - ESS - that is 
parameterized on the surface of a three-dimensional spatial structure coronary artery trees - using effective visual encodings.

In collaboration with doctors and researchers in cardiovascular imaging and applied physics, we set out to investigate the effectiveness of different visualization strategies for this problem. We first conducted an informal qualitative user study with our domain experts to develop a task taxonomy and determine their current visualization practices and needs. During this process we developed HemoVis, a novel 2D tree diagram representation that presents all the data at once while still encoding pertinent anatomical information such as vessel circumference and branch structure (Fig. 1, B). Despite positive feedback about HemoVis, we encountered resistance from users to work only with the $2 \mathrm{D}$ representation and to use a diverging color map that emphasizes features in the data more effectively. To investigate these issues we conducted a formal quantitative user study with medical domain experts to evaluate the effectiveness of $2 \mathrm{D}$ versus $3 \mathrm{D}$ representations, and the effect color has on task completion performance.

The first contribution of this paper is a task taxonomy for hemodynamics that is based on a qualitative user study with medical experts. Guided by this task analysis, our second contribution is the design of HemoVis, an interactive visualization application for heart disease diagnosis that uses a novel 2D projection and layout for artery trees. The third and main contribution of the paper is a quantitative user study with domain experts that shows statistically significant results demonstrating that our 2D representations are more accurate and efficient than $3 \mathrm{D}$ visualizations. In addition, the performance in 3D drops with an increase in the complexity of what is being examined while the 2D representation is insensitive to it. Our study also shows that a perceptually appropriate color map leads to fewer diagnostic mistakes than a rainbow color map both in $2 \mathrm{D}$ and in $3 \mathrm{D}$, and that task completion times were nearly twice as slow with the rainbow color map in $3 \mathrm{D}$. To the best of our knowledge, this is the first quantitative evaluation of the effect of spatial and color encodings for a domain task with medical professionals and real patient data.

\section{Related Work}

Vessel visualization: Vessels or branched systems are primarily visualized with $3 \mathrm{D}$ representations $[7,13,28,31]$ with scalar quantities such as ESS mapped onto the surface using color encodings [12]. In 2D, a common technique is to generate a Curved Planar Reformation (CPR) visualization of the vascular structure, where an image is generated by taking slices along a generated centerline and displaying the intensity units $[15,44]$. A drawback of CPR is that it provides limited information about the artery wall, thus not giving a clear indication of how wide or narrow the vessel is at any particular point nor convey ESS data for the entire artery. There are also other 2D projection techniques all with the goal of flattening the structure into a single view $[39,51,52]$. 3D representations have the problem of occlusion that does not allow a doctor or researcher to see all the data simultaneously. Techniques have been developed to improve 3D representations using visual cues such as shadows and transparency to improve spatial acuity in 3D [20,34] or to include 2D data [45]. In our 2D representation, all the necessary data including ESS is displayed for a medical practitioner to assess the severity of disease within an anatomical frame of reference.

2D vs. 3D visualizations: Formal evaluations are a valuable measure to determine the effectiveness of visual representations and data encodings $[18,30]$. A variety of case studies and formal user studies have demonstrated that 2D data encodings and representations are generally more effective than $3 \mathrm{D}$ for tasks involving spatial memory, spatial identification, and precision $[9,48]$. Excellent examples exist in the realms of vector field $[11,21,25]$ and geospatial visualization $[2,4,17,19,29]$. Although $2 \mathrm{D}$ is typically more effective, there are strategies to improve 3D performance, such as using occlusion and perspective $[42,50]$ or stereographic displays [47]. Our work investigates the effectiveness of 2D versus 3D artery representations in the context of heart disease diagnosis by medical experts.
Color map evaluations: Choosing the appropriate color map is essential for the effective display and analysis of quantitative data. Based on fundamental human perceptual principles and the type of data being displayed (sequential, diverging, or categorical), there are formal and systematic ways to make an appropriate color choice based on the task at hand $[14,27,33,38,46]$. Based on laboratory user studies, specific guidelines are available for the effective design of color maps $[32,36,49]$. A particular color map of interest is the rainbow map which, despite being a favorite color map across the sciences [5], is poorly suited for most data tasks and can prove misleading since it is not perceptually ordered and isoluminant [5, 23, 38, 37, 49]. Quantitative studies confirm these facts $[16,35]$ and propose better ways to design color maps and discern when isoluminant maps are suitable. Despite this general body of knowledge, there has been little study on the effects of the color map within a real-life domain application. We present a quantitative evaluation of the rainbow map's effects on task accuracy and efficiency by domain experts within a real domain application.

\section{SCIENTIFIC BACKGROUND}

Atherosclerosis, the disease focused on in this research, occurs when plaque forms in the arterial wall, causing possible obstruction of blood flow as well as changes in the outer dimension of the artery. Sites of plaque deposit (atherosclerotic lesions) form where the endothelial cells that line the arterial wall exhibit increased inflammation and permeability to lipid molecules such as LDL (i.e., bad cholesterol). Over time these plaques can either become a low-risk type that is quite large and causes a narrowing of the artery (stenosis), or a highrisk type that can rupture, potentially causing a heart attack. These high-risk deposits are not detectable with conventional imaging. In the United States, approximately 300,000 deaths per year occur from coronary artery disease and $\sim 60 \%$ are caused by rupture of these highrisk plaques [10]. However, recent research has shown that areas of low endothelial shear stress (ESS), i.e., the frictional force of blood on the artery wall, stimulate the development of these high-risk lesions $[8,43]$, and that these lesions primarily appear where there is disturbed flow, e.g., at artery bifurcations, bends, and regions of increased diameter. Thus ESS is a powerful indicator of plaque formation and disease progression.

However, it is impossible to directly measure ESS in vivo for an entire arterial tree. As a consequence, one needs to rely on blood flow simulations to calculate a patient's ESS based on their artery geometries. Combining this blood flow simulation with a patient's $3 \mathrm{D}$ reconstruction of their coronary arteries allows doctors to detect areas of low ESS, identify plaque sites non-invasively, and take preventative measures before a heart attack occurs [40]. In order to have ecological validity and develop visualizations specifically targeted at the most important diagnostic tasks, we conducted a formative qualitative user study to determine a user's tasks, what data needs to be visualized to perform these tasks, and what are the best ways to visualize the required data.

\section{Formative Qualitative User Study}

\subsection{Overview \& Logistics}

The first goal of this study was to characterize the medical and research problems being addressed by the participants and their specific domain tasks related to atherosclerosis. This was achieved by conducting a series of semi-structured interviews with 10 medical doctors and researchers representing the potential future users of such data. All the participants were interviewed at and affiliated with Brigham and Women's Hospital (Boston, MA). In an attempt to cover as broad an audience as possible, study participants ranged in age, gender, experience level, education background, job seniority, clinical versus research focus, and department (radiology versus cardiology).

Each participant was interviewed and asked the same set of questions to gather sufficient information on their background and experience, research interests, knowledge of hemodynamics, and their current workflow goals and tasks. Each participant was then shown a 
Table 1. Domain tasks, broken down by clinical versus research focus, of participants based on a formative qualitative user study.

\begin{tabular}{|c|l|c|c|}
\hline ID & Task & Clinical & Research \\
\hline \hline 1 & Identify stenosis or blockage & $\mathrm{X}$ & $\mathrm{X}$ \\
\hline 2 & Identify regions of low ESS & $\mathrm{X}$ & $\mathrm{X}$ \\
\hline 3 & $\begin{array}{l}\text { View all ESS data for heteroge- } \\
\text { neous patterns }\end{array}$ & $\mathrm{X}$ & $\mathrm{X}$ \\
\hline 4 & $\begin{array}{l}\text { Study blood flow (velocity) pat- } \\
\text { terns }\end{array}$ & $\mathrm{X}$ \\
\hline 5 & $\begin{array}{l}\text { Identify regions of blood recircu- } \\
\text { lation }\end{array}$ & $\mathrm{X}$ \\
\hline 6 & $\begin{array}{l}\text { Investigate other physical vari- } \\
\text { ables of blood flow }\end{array}$ & $\mathrm{X}$ \\
\hline 7 & $\begin{array}{l}\text { Follow patient's disease progres- } \\
\text { sion }\end{array}$ & $\mathrm{X}$ & $\mathrm{X}$ \\
\hline
\end{tabular}

series of images covering 2D and 3D representations of ESS. The desired outcome was, based on the resulting feedback, to answer the following questions: What data should be shown to accomplish tasks of clinical importance? What are the optimal 2D representations? What are the optimal 3D representations? Should the data be encoded in 2D or 3D? And what color schemes are best to aid the individual in task completion?

\subsection{Task Taxonomy}

The participants' jobs fall into two broad categories: clinical diagnostics and fundamental research. The former represents those individuals who work on making clinical diagnoses for specific patients, and the latter represents individuals who work on investigating the fundamental causes of heart disease. Some individuals fall into both categories (e.g., doctors who split their time between medical practice and research). Table 1 represents all the domain tasks cited by participants and category of individuals that cited the task. Both categories of individuals need to accomplish the same basic set of tasks essential to the immediate diagnosis of patients based on factors that have a proven link to atherosclerosis (i.e., low ESS, artery geometry). However, those with a research focus care about further exploring the data to investigate variables or aspects not necessarily with a proven link to disease.

Each of these domain tasks can be abstracted to fundamental analytic tasks presented by Amar et al. [1]. Tasks 1 and 2 correspond to finding extrema. The clinical diagnostics study participants expounded on the need for these tasks to be accomplished in a quick and efficient manner with minimal cognitive effort for rapid diagnosis of the patient. These tasks are usually done inside or just outside a procedure room to expedite taking preventative measures (e.g., insertion of a stent). In a research setting the time pressure was lower. Task 3 corresponds to clustering of the data. In a clinical setting, this task is not as essential or of the same importance as 1 or 2, but would be necessary for diagnosis in complicated cases. Task 4-6 are about finding anomalies in the data. In these cases time is not a factor, and being able to analyze the data carefully is the primary goal. Finally, task 7 is a correlation task, requiring comparison of multiple data sets, if available for a patient, in order to follow the progression of the disease.

\subsection{D Representations}

Projections The first portion of the qualitative user study was focused on 2D projections of the arteries. This is relevant to Tasks 1-3 (see Table 1). All but one of the participants had been exposed to reading ESS values from a cylindrical projection representation of an artery, and all were familiar with ESS in general. Initially two different types of projections were shown to participants (Fig. 2 top and middle). The top image is a traditional cylindrical projection in which the length of the image is based on the length of the artery, and the width is arbitrarily chosen. The middle image represents a
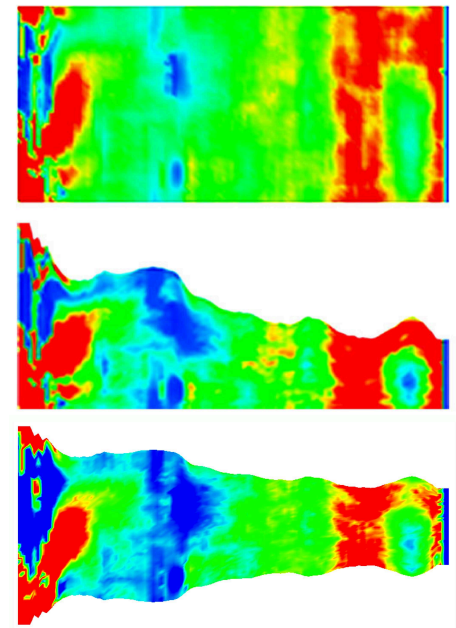

Fig. 2. The three different cylindrical projection techniques presented to users during the formative qualitative user study. Top: Traditional cylindrical projection. Middle: Projection with circumference mapped to image height above the x-axis. Bottom: Projection with circumference mapped to the width of image symmetric on centerline (preferred projection by users).

variation on this cylindrical projection in which the width equals the circumference of the artery at the cross section.

During the user study, the first two participants in the postevaluation interview commented on the middle cylindrical projection. They both said that it was slightly confusing to them and that, when viewing the image, they were mentally reflecting the image over the $\mathrm{x}$-axis to make it symmetric. Upon presenting them with the pseudocylindrical projection based on their feedback depicted in Fig. 2 (bottom), they both commented that this was a superior mapping. This projection maps the circumference of the artery at the cross section to the height, and centers it along a centerline. For the remainder of the study we presented all three projections to the participants.

All the participants preferred the pseudo-cylindrical projection map (Fig. 2 bottom). When asked whether this projection is confusing, they replied "no". Although not exactly reproducing the real geometry, the representation is able to encode enough geometric information to show the user the relationship between geometry and ESS as well as where stenoses occur. This representation is also intuitive for clinicians since the 2D mapping mimics what one would see if an artery is cut-open ("butterflied") for ex vivo studies.

Tree Diagram Layout Continuing with 2D representations, for tasks 1-3, all of the study participants were presented with a visualization of the artery tree in with each node was representative of an artery and branches indicative of bifrucations (Fig. 3). When presented with the hierarchical tree layout in Fig. 3 (top), no one had ever seen anything like it before and all except one individual responded that they greatly preferred this data representation and found it useful. Unlike CPR visualizations that only include two locations of the artery wall $[15,44]$, this tree diagram not only shows the artery structure but conveys the length and width of each vessel. The most common feedback on the tree diagram was the great benefit of being able to see all the data at once without the usual occlusion challenges found in 3D representations, and being able to display and compare multiple data sets at the same time (task 7). The two most commonly cited scenarios where this would be useful are for viewing a patient's data with the blood flow simulation adjusted to simulate a patient at rest and at heightened physical exertion (i.e., low and high flow rates), and for viewing multiple image acquisitions of a patient over time in order to view and compare the simulation output for each. This latter scenario would aid the doctor in observing the progression of disease over time. 

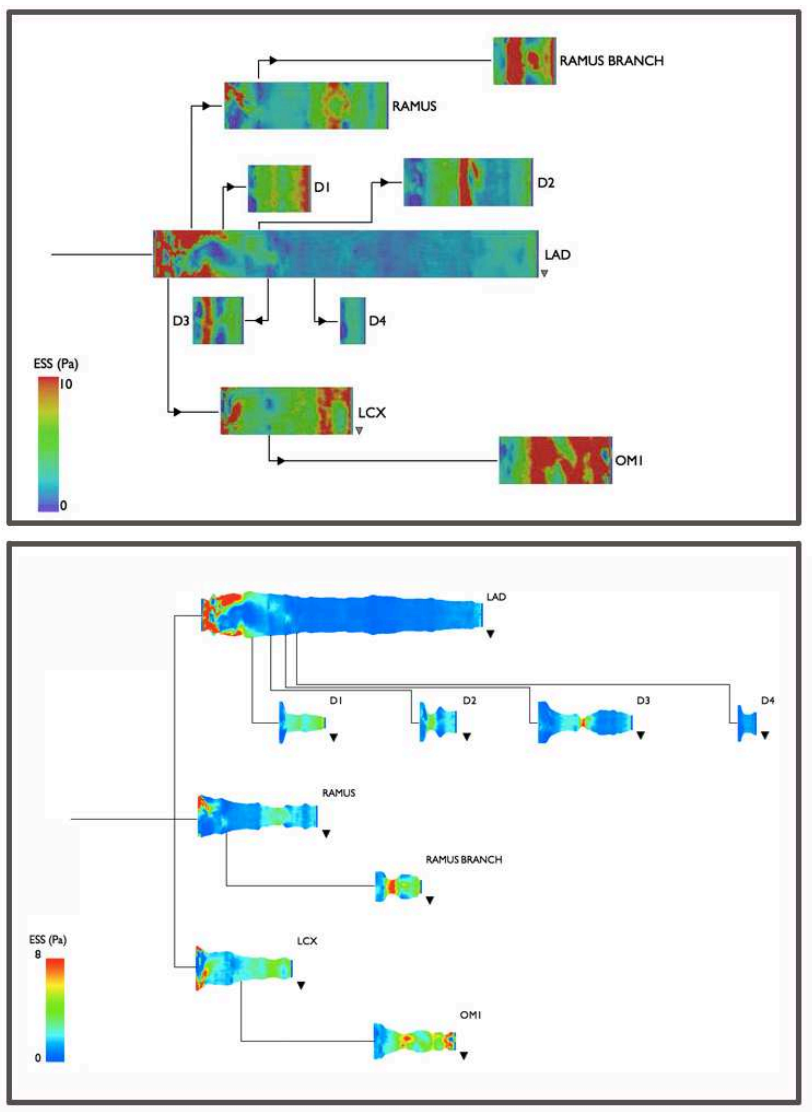

Fig. 3. Original and final tree layout schemes. Top: Initial design of tree layout. Bottom: Final tree layout based on feedback from users to make it more anatomically representative.

As shown in the initial sketch (Fig. 3 top), the study subjects were presented with some possible interactions with the tree data including the ability to open and close branches, move branches around the screen, and interactively crop the color scale. All of the participants said that closing the branches is not useful since they always want to see the other data sets and keep the data in context. Everyone also stated that being able to move or drag branches around the screen is not a good idea since they want to maintain the same static anatomical structure when viewing the data for context.

All of the user study participants, except the research staff participants without advanced degrees, noted that the tree was not accurately conveying the anatomical information. When the initial design (Fig. 3 top) was created, the branches were arranged to fit the space available on the screen. Independent of each other, the participants were asked to describe or draw what adjustments they would make to create an anatomically "correct" version of the tree. All of these individuals made the same recommendations. For the vertical arrangement, the branches should be ordered from major to minor arteries with the placement of each descending branch point below the "parent" vessel (and if descending branch "superior", i.e., branch points anatomically go upwards, place it above the parent vessel). If there are two very close major bifurcations, then present it in the tree diagram as a trifurcation. Finally, always have the tree diagram lines angled and drawn in the same direction as the blood flow (does not make sense to have blood flow "upstream") which also eliminates the need for directional arrows.

The final diagram is shown in Fig. 3 (bottom). It should also be noted that the sample data set we used for the study has a large complex branched structure and that the ESS data is high resolution compared to a typical data set. This was chosen on purpose to represent the most complicated large case available with conventional scan technol- ogy. Finally, to evaluate whether a portrait or landscape presentation was more useful, the participants where shown a tree diagram representation in each orientation. All the participants preferred the landscape orientation depicted in Fig. 3 (bottom) since it was intuitive to "read" the visualization and flow direction from left to right, and comparisons were easier for multiple data sets when stacked vertically.

\subsection{D Representations}

The next portion of the formative qualitative user study was focused on the 3D display of ESS data, and which of 2D or 3D display techniques may be better for the completion of the domain tasks. All of the participants had previously seen 3D representations of ESS as a 3D surface with ESS mapped in color onto the exterior (see Fig. 1, C). Mapping ESS in 3D is useful since it maintains the true geometry and anatomy a doctor is accustomed to seeing, and makes it easier to translate the knowledge back to a surgical setting. A common practice, cited by the study participants and visible in the literature, is to choose standard viewing angles that a doctor or surgeon would be interested in. However, to view all the data effectively at the same time requires interactivity or animated rotation. 2D projections of ESS, such as those discussed in Secion 4.3, have the advantage of being able to show all the data without occlusion, to display all the data in a static state, and to easily compare multiple data sets.

\subsection{Color}

A portion of the qualitative study was devoted to determing the best color schemes to encode scalar ESS data in either 2D or 3D. Except for this portion of the study, all images presented during the study were created using a rainbow color scheme. Rainbow is the standard color map used in the medical literature, and we did not want the participants to be distracted by unusual colors when asking about spatial data encoding techniques.

A total of eight carefully designed simple color schemes were presented to the users (see Fig. 4). The priority was designing a scheme that brought out the data structure to accomplish tasks $1-3$, particularly the data which is not as visible when viewed with the standard rainbow map [22]. Four of the color schemes are based on diverging color tables from Cynthia Brewer's ColorBrewer [6]. The other two diverging color schemes were designed based on the concept of luminance being easy to read with it encoding the scalar ESS value and with chroma only highlighting the highest or lowest ESS values. Also included was a simple luminance scheme with no color. The color schemes presented to the study participants are shown in Fig. 4: a rainbow map [A], a desaturated rainbow map with yellow at the mid-point (rather than the standard green) [B], a diverging map from blue to red with white at the divergence point [C], a diverging map from blue to red with cream at the divergence point [D], a sequential greyscale map [E], a diverging map from red to black with white at the divergence point $[\mathrm{F}]$, a diverging map from blue to black with white at the divergence point $[\mathrm{G}]$, and a diverging map from purple to orange with white at the divergence point $[\mathrm{H}]$.

Every study participant except one said that they liked the rainbow scheme the best. The reasons for this are that it is what they are "used to seeing", the colors are more saturated than those in the other scales making it "easier to see", it is the "most aesthetically pleasing" of the choices, and that it is the "easiest to directly determine the numerical value" of the ESS based on matching the color to the scale. However, the participants were astute and saw, after viewing all the color maps, that red is the most eye-catching as it has a "pop-out" effect [3]. Multiple study participants acknowledged that their primary task is identifying regions of low ESS so it would make sense to have the areas of concern be red. Thus they suggested a more useful version of the rainbow scale would be to invert it, so that red indicates low ESS and blue high ESS.

Examining the non-rainbow schemes, the simple grayscale shows great detail and subtleties in the ESS data, especially compared to the rainbow scale. However, most of the participants objected to this scheme citing that when they see a black-and-white image they assume the data is raw radiological imagery (e.g., x-ray, MRI, etc.) and 

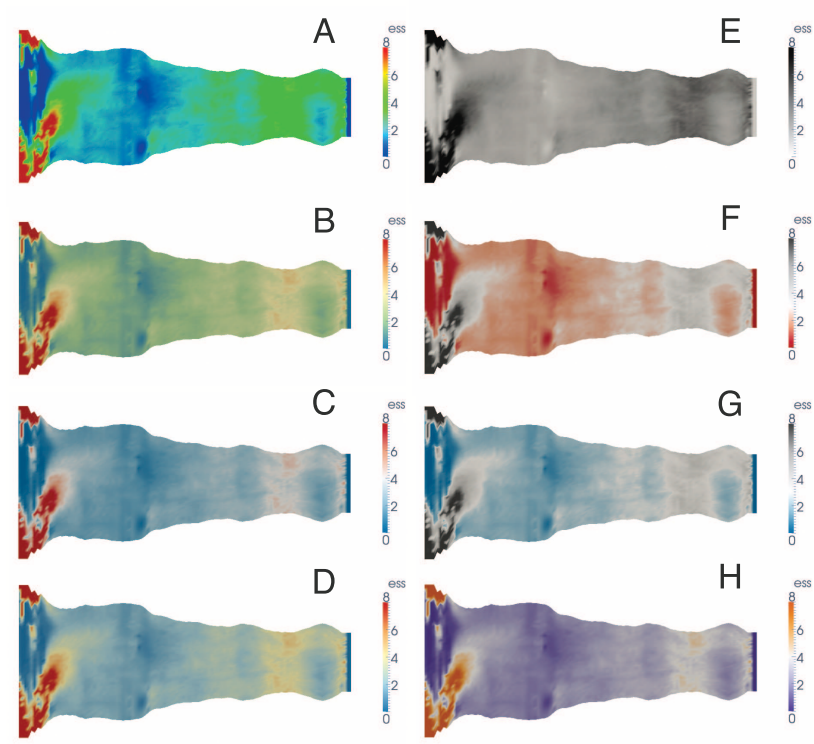

Fig. 4. Color schemes presented during the qualitative user study. The rainbow scheme $(A)$ was preferred by most since it is what they are accustomed to viewing. The next most popular scheme was the redblack diverging scale $(F)$. The grayscale image (E) was unanimously disliked since participants assume black-and-white images to be raw radiological data, while color indicates that the data has been processed or simulated.

not simulated data. When they see a false-color scale, they immediately make the assumption that it is processed and/or simulated data and not raw imagery. Thus it is important to include some form of color when mapping data like ESS to avoid confusion.

Of the non-rainbow schemes, the one study participants liked the best was the red-to-black diverging scale. They felt it did the best job of grabbing their attention to the highlighted areas of extreme ESS and showing the data structure. Although some of these users had pointed out that a pure luminance scale with no color usually indicates raw imaging data, none of them complained or mentioned this when picking the red-to-black scale. Finally, one user liked the diverging red-to-blue schemes the best. Also, a number of participants gave positive comments during this color design portion of the study, acknowledging that they could see more structure in the data when using a non-rainbow scheme, and that they would consider using a diverging color scheme instead in their own data analysis.

In summary, the key take-aways and lessons learned during the formative qualitative user study are to keep the data representation as anatomically correct as possible (i.e., choice of 2D projection and tree diagram layout); that a 2D data display is more effective for data analysis and for comparing multiple data sets; that the best color choice is a diverging color scheme utilizing red to highlight the regions of greatest interest; and that a pure black-and-white color scheme should be avoided since users associate it with raw radiological data. As will be discussed in the following section, we applied all these principles in the development and design of HemoVis.

\section{HemoVis}

Using an iterative task-driven design based on our formative qualitative user study, we developed a 2D interactive visualization called HemoVis ${ }^{1}$ (Figs. 5 \& 6). The design is based on the qualitative evaluation with additional feedback from select users and the task taxonomy with a focus on the tasks that are most relevant for both clinical and research settings (1-3 \& 7 from Table 1) as described in Sec. 4.2.

HemoVis has two viewing modes: tree (Fig. 5) and individual (Fig. 6). In tree mode, a tree diagram of the arterial system is pre-

\footnotetext{
${ }^{1}$ Available online at http://www.seas.harvard.edu/ borkin/HemoVis
}

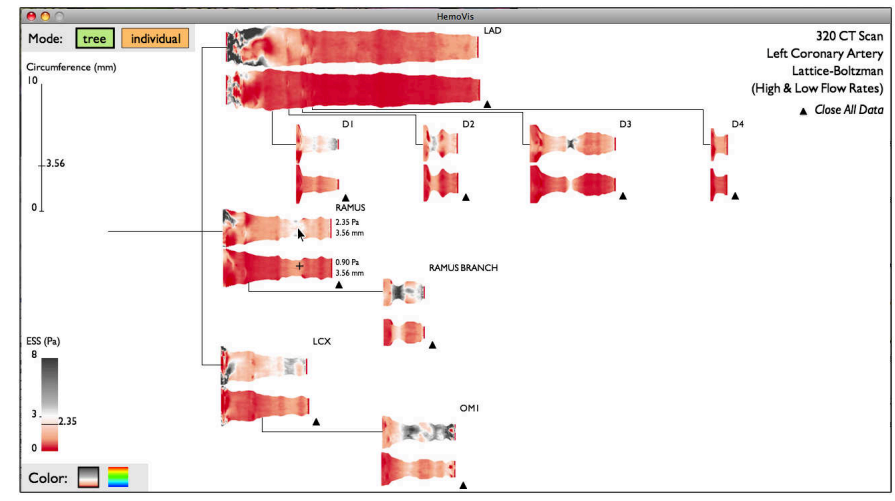

Fig. 5. HemoVis in the "tree" mode displaying a patient's left coronary artery tree with color mapped to ESS.

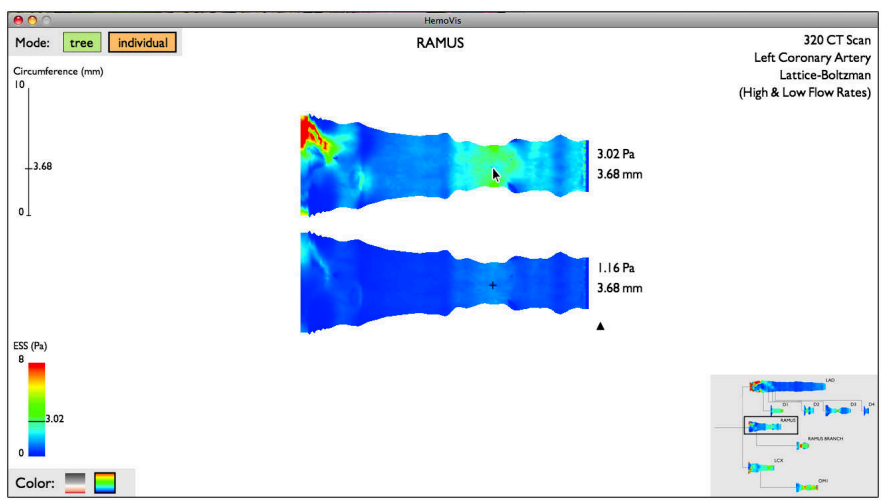

Fig. 6. HemoVis in the "individual" mode displaying a single artery with small tree diagram for navigation in the lower right.

sented in which each node is representative of an artery and each line segment representing a bifrucation. Each artery is displayed using the 2D pseudo-cylindrical projection discussed in Sec. 4.3, is labeled with its anatomical name, and has its ESS values encoded with color. The color and size scales are displayed to the left of the tree diagram. The upper right of the screen displays the relevant metadata for the particular data set. The interaction techniques implemented are based on expert feedback from the qualitative study and follow-up discussions. The user is able to simultaneously view additional simulation data sets for the same patient by clicking the small triangles. A user can mouseover the images to display exact quantitative ESS and circumference measurements. If the second data set is open for a particular artery, then a cross-hair cursor will appear on the image opposite from the mouse cursor indicating the equivalent position. One can also change the desired color mapping by selecting one of the colored boxes in the lower left corner. To switch modes, there are two mode buttons in the upper left. In the alternate individual mode, only one artery is displayed at a time allowing the user to take care at studying particular arteries in high resolution. In this mode there is also a small non-interactive version of the entire coronary tree in the lower right corner to help keep the displayed artery in context as well as be used to navigate the branches by clicking them. HemoVis is implemented in Processing 2 .

The data for both our qualitative and quantitative (Sec. 6) user studies comes from the Multiscale Hemodynamics Project ${ }^{3}$. The patients' coronary geometries are obtained from CTA data acquired with a 320 detector row Toshiba AquilionONE scanner. The data is 4D from a series of cardiac cycles which is then registered into a single volume. The data is then semi-automatically segmented using Vitrea (Vital Im-

\footnotetext{
${ }^{2}$ http://www.processing.org

${ }^{3}$ http://hemo.seas.harvard.edu
} 
ages Inc). The end result is a series of 3D surfaces of the heart and coronary arteries. These geometries are then loaded into MUPHY, a multi-physics and multi-scale code combining Molecular Dynamics (MD) with a Lattice Boltzmann (LB) method, to model the blood flow through the static geometries [26]. The simulation was run using a parallel implementation on Harvard's IBM BlueGene/L. The result is 3D data of the simulated blood flow and associated properties including ESS.

However, despite positive feedback from users and the domain expert driven iterative design, many potential users were reluctant to try the prototype because they were not convinced it was really better than a 3D representation. Also, despite expert acknowledgment during the formative qualitative study's section on color choice that some of the non-rainbow schemes did an excellent job of displaying features in their data and presenting users with background literature on the rainbow color map (see Sec. 2), the users were reluctant to choose an alternative color map to rainbow. Additionally we wanted to quantitatively investigate, with this real world example, what effect data representation and color encoding has on task performance. As a result, we decided to conduct a formal quantitative user study to evaluate if a 2D representation is more effective than $3 \mathrm{D}$ and if color can effect how one perceives ESS features.

\section{Quantitative User Study}

We conducted a formal quantitative user study to determine whether a 2D or 3D data representation of ESS was more effective and efficient for diagnosing a patient's coronary artery disease. We also wanted to see if there were quantitatively measurable performance effects based on the color scheme utilized, specifically the rainbow and the diverging red/black color maps. Having an effective visualization is important in making an accurate diagnosis, but having an efficient visualization is also important in order to allow a medical professional to take rapid preventative measures if needed as well as increasing overall hospital efficiency. To maintain high external validity of our results for this domain, we worked with medical professionals and real patient data.

\subsection{Hypotheses}

Our hypotheses entering the user study were:

H.1 Compared to a 3D representation, a 2D data representation will result in fewer diagnostic errors and faster performance.

H.2 A non-rainbow color map, specifically a diverging color map, will result in fewer diagnostic errors and faster performance than a rainbow color map.

\subsection{Participants and Apparatus}

In order to have a large number of medically literate participants in the study, we chose to use medical students. These participants all had the basic medical expert training and knowledge of cardiovascular disease and anatomy and did, therefore, have the necessary expertise to fully understand and complete the tasks presented in the study. Participants had no prior bias towards any specific ESS visualization because the non-invasive diagnostic technique being presented here is not yet a part of standard clinical practice.

Twenty-one Harvard Medical students participated in the study. This included 12 women and 9 men, with a spread of $1^{\text {st }}$ through $4^{\text {th }}$ year students. All participants reported having normal color vision and were additionally checked at the beginning of their session using a standard Ishihara pseudo-isochromatic plate series for detection of protan/deutan (i.e., red/green) and tritan (i.e., blue/yellow) color vision deficiencies. Each participant was monetarily compensated for their time at the end of their session.

All study sessions were conducted in the same room with identical lighting, and on the same MacBook Pro 15" laptop. Participants where offered the choice of a wireless mouse or trackpad based on which they felt more comfortable using; every participant chose the wireless mouse. The 2D representations were displayed using Preview, and the $3 \mathrm{D}$ representations were displayed using Paraview. Audio, video screen capture, and mouse clicks and movements were all recorded with Screenflick.

\subsection{Tasks and Procedure}

The study session for each participant started with the color vision test, followed by a basic survey to obtain demographic information and to assess their knowledge of both heart disease and fluid dynamics. The participants were then provided with two pages of background information giving a brief overview of the project and the new noninvasive diagnostic tool being evaluated. Next the participants where given instructions for their task ("identify all low ESS regions") and shown a series of images (see Fig. 1 (B) for sample 2D and (D) for equivalent $3 \mathrm{D}$ representation) for them to perform the task on with a survey in-between each image to gauge their confidence levels. The session concluded with verbal questions and feedback.

During the main part of the experiment, each participant was shown on a LCD screen a series of 8 images with the first 2 serving as a training tasks. The images alternated between $2 \mathrm{D}$ and $3 \mathrm{D}$ representations to minimize difference in learning effects between the two representations, and the images included an evenly distributed mix of left and right coronary artery trees (since the left and right sides have slightly different anatomical structures and complexities as demonstrated in the $3 \mathrm{D}$ representations (C and D) of Fig. 1 ). The data used in the 3rd and 4th images shown to the participant were also used in the 7 th and 8 th images but using the alternate $2 \mathrm{D} / 3 \mathrm{D}$ representation to allow for a larger number of measurements per participant.

The participant's task (which was explained in both printed and verbal instructions with annotated sample visualizations) was to identify all the low ESS regions in each image. In both 2D and 3D conditions, a person could indicate small regions by clicking on them with the left mouse button, while larger regions could be marked by encircling them with the mouse cursor. These actions left no visible mark on the image, but were recorded by our software for post-experimental analysis. In $3 \mathrm{D}$, the participant could arbitrarily rotate the model. Based on results from our pilot run of this study, we did not enable zooming because it did not improve the diagnostic accuracy, but frequently caused participants to become disoriented, losing track of which parts of the image they had examined and which they had not.

After each image, the participants filled-out a questionnaire where, based on the task they had just completed, they were asked to respond on a 7 -point Likert scale ( $1=$ strongly disagree, $7=$ strongly agree $)$ to four statements: "I found it easy to identify low shear stress regions", "I was able to perform the task efficiently", "I am confident I found all the low shear stress regions", and "I am confident all the places I marked are really low shear stress."

At the end of the session, each participant was verbally asked which visualization style (i.e., 2D or 3D) they preferred and why, and asked whether they had other comments, questions, or feedback. Each session lasted approximately 40 minutes.

\subsection{Experimental Design \& Analysis}

The study was a mixed between- and within-subject design with the following factors and levels:

- dimensionality of representation (2D or 3D);

- color mapping (rainbow or diverging).

Dimensionality of representation was a within-subject factor and color mapping was a between-subject factor.

Our dependent measures were the fraction of low ESS regions identified, the number of false positives (i.e., non-low ESS regions identified as ESS), and the time to complete a diagnosis. Because the time to complete a diagnosis was impacted by the number of low ESS regions a participant identified in each image as well as the total number of low ESS regions present in each image, we additionally compared participants' performance in terms of the average amount of time taken to identify a low ESS region (i.e., total time spent on an image divided by the number of low ESS regions identified). 


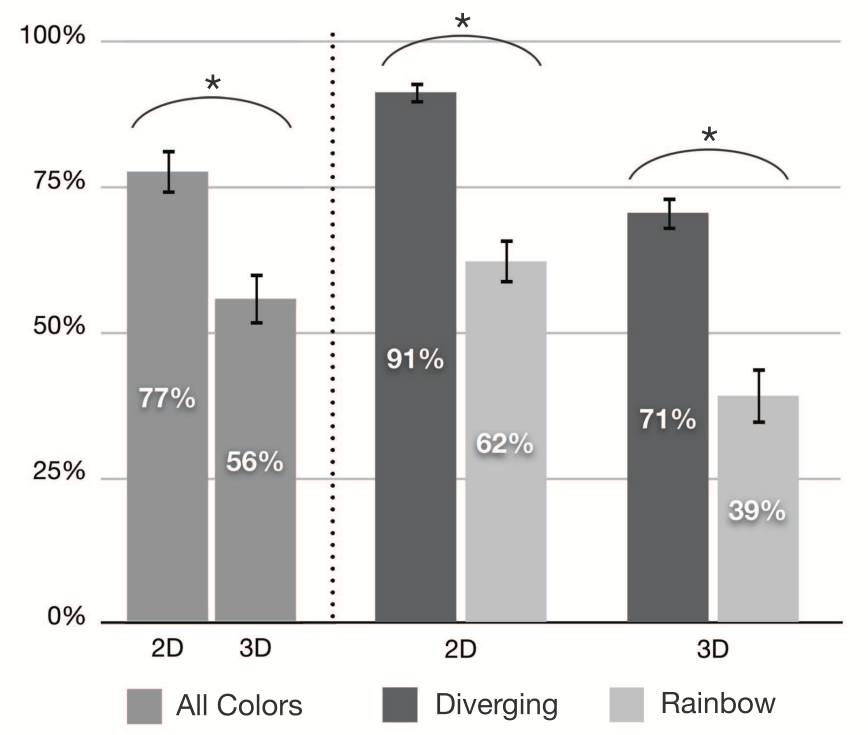

Fig. 7. Average percent of low ESS regions identified broken down by $2 \mathrm{D}$ and $3 \mathrm{D}$ representation, and color. Error bars correspond to the standard error and the asterisks indicate results of statistical significance. Participants were more accurate in $2 \mathrm{D}$ and when using the diverging color map.

To generate the two accuracy-related measures, each participant's responses (regions they encircled with the mouse cursor or clicked on) were compared against answer keys generated by cardiovascular imaging specialists. After each trial, we also collected four subjective measures as described in the previous section.

Half of the participants started with a 2D representation and half with a 3D representation. Similarly, half of the participants started with a left coronary artery tree and half started with a right. The orderings of data sets (three hearts) were counterbalanced using Latin Square design. Genders were balanced between the two color mapping conditions and between tasks starting with $2 \mathrm{D}$ and $3 \mathrm{D}$ representations.

The time to complete a diagnosis followed a lognormal distribution. We log-transformed these data as is common practice and analyzed it with a t-test. For the remaining measures, we used nonparametric tests: the Wilcoxon signed rank test for within-subject comparisons, and the Mann-Whitney U test for between-subject comparisons. To guard against Type I errors, we applied the Holm's sequentially-rejective Bonferroni procedure [41] to the analyses of the subjective responses and to the additional analyses that did not correspond directly to our two stated hypotheses.

Because of the substantial qualitative differences between the 2D and $3 \mathrm{D}$ conditions, we analyzed the effects of color separately for each of these two conditions.

\subsection{Results}

Preliminaries A contrast analysis of the fraction of low ESS regions identified across the 6 test tasks revealed no significant learning effects $(Z=-21.5, p=0.47)^{4}$. That is, the participants' ability to correctly identify low ESS regions did not change significantly throughout the experiment. We thus include results from all 6 tasks in our subsequent analyses.

Accuracy We observed a main effect of the dimensionality of representation on the fraction of low ESS regions identified $(Z=$ $-115.5, p<0.001$ ): participants correctly identified $77 \%$ of low ESS regions in $2 \mathrm{D}$ images, but only $56 \%$ in $3 \mathrm{D}$ (see Fig. 7). In both $2 \mathrm{D}$

\footnotetext{
${ }^{4} \mathrm{Z}=\mathrm{z}$-score for the Wilcoxon signed rank test, $\mathrm{p}=\mathrm{p}$-value.
}

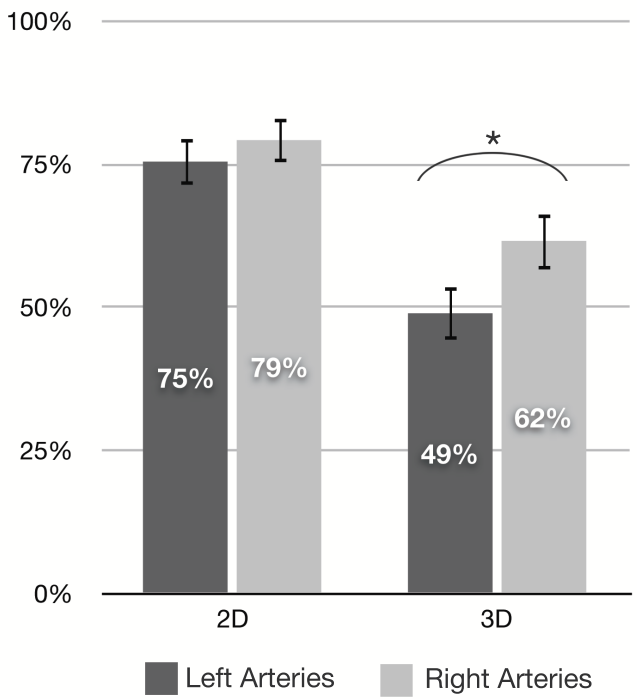

Fig. 8. Average percent of low ESS regions identified broken down by $2 \mathrm{D}$ and $3 \mathrm{D}$ representation, and left and right artery systems. Error bars correspond to the standard error and the asterisks indicate results of statistical significance. In 3D, users were less accurate identifying regions in the most complex data sets (i.e., left artery systems). Whereas in 2D, performance was the same regardless of task complexity.

and 3D conditions, we also observed significant effects of color mapping on the fraction of low ESS regions identified. For 2D images, participants in the diverging condition found $\sim 47 \%$ more low ESS regions than the participants in the rainbow condition $(U=1, p<0.001$, $r=0.83)^{5}$. For $3 \mathrm{D}$ images, the diverging color map resulted in an $\sim 82 \%$ improvement over the rainbow color map $(U=7, p=0.001$, $r=0.74)$.

For the top performing combination ( $2 \mathrm{D}$ with non-rainbow), the low ESS regions that were not identified by participants were generally the smallest in area of all the regions in a given data set. These regions were also very close to the diverging point in the color map bordering between "low ESS" and "normal". In the other conditions, there was no observed regularity in the low ESS regions missed.

We observed a negligible number of false positives (only 6 instances across all users). These false positives occurred in both color schemes, but all occurred only in 3D representations.

We additionally examined the difference in accuracy between the left and right coronary artery branches as shown in Fig. 8. The left branch systems are more complex due to additional bifurcations inherent to the anatomy. In our data sets, the left artery systems ranged from 7 to 10 branches $(M=8)^{6}$ and the right artery systems ranged from 4 to 7 branches $(M=6)$. On average the left artery systems had 25 low ESS regions and the right artery systems 17 low ESS regions. There was no significant difference in accuracy between these data types in $2 \mathrm{D}(Z=-1.57, p=0.117)$. However, in 3D participants were significantly less accurate when identifying regions in left artery systems than in right systems $(Z=-3.35, p=0.001)$. This provides evidence that in $3 \mathrm{D}$ the performance accuracy decreases with increased data complexity.

Efficiency On average, participants spent less time per image in the $2 \mathrm{D}$ condition $(\mathrm{M}=56$ seconds) than in the $3 \mathrm{D}$ condition $(\mathrm{M}=$ 73 seconds) and this difference was statistically significant $(t(21)=$ $-2.52, p=0.021)^{7}$ (see Fig 9). In the 2D condition, we also observed

\footnotetext{
${ }^{5} \mathrm{U}=$ Mann-Whitney U measure, $\mathrm{p}=\mathrm{p}$-value, $\mathrm{r}=\mathrm{Z} / \sqrt{N}$

${ }^{6} \mathrm{M}=$ mean.

${ }^{7} \mathrm{t}(\#)=\mathrm{t}$-test with \# of participants
} 


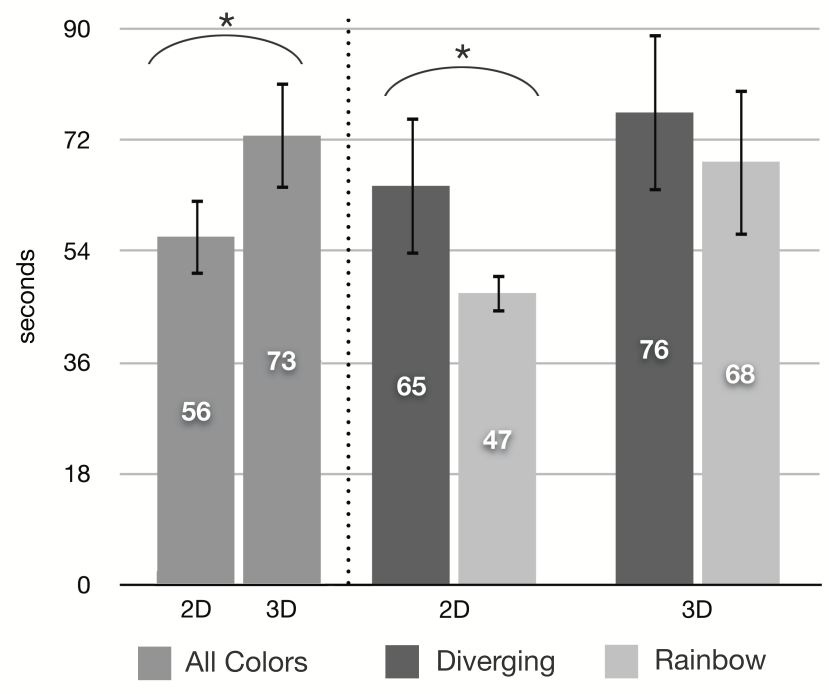

Fig. 9. Average total time spent on each image broken down by $2 \mathrm{D}$ and 3D representation, and color. Error bars correspond to the standard error and the asterisks indicate results of statistical significance. Participants completed tasks more quickly in 2D than 3D.

a significant effect of color mapping on the average task completion time $(t(21)=-1.57, p=0.013)$ : participants spent less time on images using the rainbow color map ( $M=47$ seconds) than on images using the diverging color map ( $\mathrm{M}=65$ seconds). We saw no such effect in the 3D condition $(t(21)=-0.351, p=0.741)$. Even though participants completed 2D images more quickly with the rainbow color map, they had poor accuracy as described in the previous section.

Therefore, we next look at the average amount of time taken to identify a low ESS region (i.e., total time spent on an image divided by the number of low ESS regions identified). As illustrated in Fig. 10, there is a significant difference between participants' performance in $2 \mathrm{D}$ and $3 \mathrm{D}(Z=115.5, p<0.001)$ with participants identifying regions more quickly in $2 \mathrm{D}(\mathrm{M}=2.5$ seconds per region) than in $3 \mathrm{D}(\mathrm{M}=7.8 \mathrm{sec}-$ onds per region). There is no significant effect of color mapping in 2D with respect to this measure $(U=44, p=0.439, r=0.169)$ indicating that the utility and effectiveness of the $2 \mathrm{D}$ representation outweighs the effect of color in regards to rate of identifying regions. However, we did observe a significant effect of color mapping in $3 \mathrm{D}(U=18$, $p=0.009, r=0.567)$ with participants identifying regions approximately twice as fast with the diverging color map. Thus the effect of the rainbow color map on task efficiency has a greater impact in 3D than in 2D.

Subjective Responses When examining the subjective statements, statistically significant differences were observed between the $2 \mathrm{D}$ and $3 \mathrm{D}$ representations.

As shown in Fig. 11, on a 7-point Likert scale $(1=$ strongly disagree, $7=$ strongly agree) participants indicated that on average it was easier to identify low ESS regions in 2D than in 3D $(Z=-75.5$, $p<0.001)$. They also reported that it was more efficient to identify regions in $2 \mathrm{D}(Z=-72.5, p<0.001)$, and that they were more confident they found all the low ESS regions in $2 \mathrm{D}(Z=-68.0, p<0.001)$. There was no statistically significant effect of dimensionality of presentation on participants' confidence that what they marked as low ESS were in fact really a low ESS regions $(Z=-33.5, p=0.146)$. This is consistent with their actual performance: as reported earlier, we observed very few false positives throughout the study.

We observed no statistically significant effects of color scheme on any of the participants' subjective responses. This indicates that the participants thought they did well using the rainbow color map even

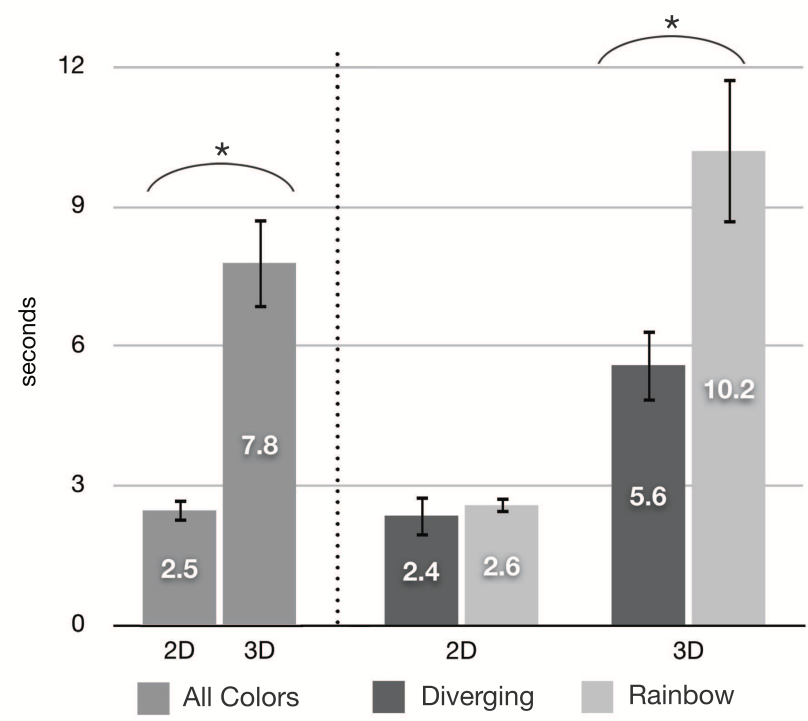

Fig. 10. Average rates of seconds per region to identify broken down by $2 \mathrm{D}$ and $3 \mathrm{D}$ representation, and color. Error bars correspond to the standard error and the asterisks indicate results of statistical significance. Participants were more efficient in 2D, and in 3D there was a significant difference in participant performance between color schemes.

when in reality they did not perform as well as the participants who used the diverging color map.

\subsection{Discussion}

The results fully support our first hypothesis: participants missed fewer low ESS regions in 2D than in 3D and they completed the tasks more quickly (both in terms of total time and when comparing times spent per low ESS region identified).

This was also reflected in the verbal question portion at the end of the study sessions in which 18 out of the 21 participants said they preferred the 2D representation citing it was "easier", "more efficient", and "better for viewing the data since all the data is visible at once". Of the 3 participants who preferred the 3D representation, 2 of the participants verbally acknowledged that the $2 \mathrm{D}$ visualization was better and more efficient for completing the task but chose 3D as their "preferred" representation due to aesthetics.

The results also partially support our second hypothesis: in both 2D and $3 \mathrm{D}$ conditions participants who were presented with the data using the diverging color scheme made fewer diagnostic mistakes than those who saw the same data presented in the rainbow color scheme. The efficiency results are less equivocal: even though the rainbow color scheme resulted in faster total completion times in $2 \mathrm{D}$, controlling for the number of low ESS regions identified, we saw no performance differences due to color mapping in 2D, but in 3D we observed participants being nearly twice as slow on a per region basis with the rainbow color mapping than with the diverging.

Part of the reason why the 2D representation is efficient is because people are able to easily "read" across the image and mark regions in a systematic manner. We concluded this based on the observed order in which participants identified low ESS regions and statements from participants during the verbal feedback section. In contrast, there is no obvious strategy for "reading" across the 3D representation. The $3 \mathrm{D}$ visualization also requires one to rotate and interact with the image, thus it takes longer for someone to view all the data. In addition to participants verbally complaining about the added interaction, participants had a difficult time remembering where they had previously identified a region of low ESS in the 3D representation. Thus in order to make the $3 \mathrm{D}$ visualization more effective, one would need to develop a good "mark-up" strategy such that a person knows what re- 


\begin{tabular}{|c|c|c|c|c|c|c|c|c|c|}
\hline \multirow[b]{2}{*}{ Statement: } & $2 D$ & 3D & \multirow[b]{2}{*}{ 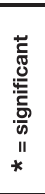 } & \multicolumn{2}{|c|}{ 2D } & \multirow[b]{2}{*}{ 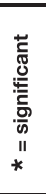 } & \multicolumn{2}{|c|}{ 3D } & \\
\hline & \multicolumn{2}{|c|}{ (both color maps) } & & 产 & 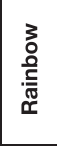 & & $\begin{array}{l}\stackrel{0}{5} \\
\frac{5}{0} \\
\stackrel{2}{0}\end{array}$ & 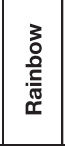 & 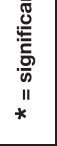 \\
\hline $\begin{array}{l}\text { I found it easy to } \\
\text { identify low shear } \\
\text { stress regions. }\end{array}$ & 6.30 & 5.35 & * & 6.33 & 6.27 & & 5.48 & 5.20 & \\
\hline $\begin{array}{l}\text { I was able to } \\
\text { perform the task } \\
\text { efficiently. }\end{array}$ & 6.27 & 5.30 & * & 6.21 & 6.33 & & 5.55 & 5.03 & \\
\hline $\begin{array}{l}\text { I am confident I } \\
\text { found all the low } \\
\text { shear stress } \\
\text { regions. }\end{array}$ & 6.11 & 5.17 & * & 6.30 & 5.90 & & 5.55 & 4.77 & \\
\hline $\begin{array}{l}\text { I am confident all } \\
\text { the places I marked } \\
\text { are really low shear } \\
\text { stress. }\end{array}$ & 5.84 & 5.63 & & 5.91 & 5.77 & & 5.85 & 5.40 & \\
\hline
\end{tabular}

Fig. 11. Averages of subjective responses broken down by $2 \mathrm{D}$ and $3 \mathrm{D}$ representation, and color. The four statements are rated on a 7-point Likert scale ( 1 = strongly disagree, 7 = strongly agree), and the asterisks indicate results of statistical significance. Participants felt it was easier and faster in 2D, and they felt more confident in 2D.

gions they have already identified or arteries already inspected. The 2D representation also makes it easier to identify regions of low ESS by easily exposing complex 3D features, such as artery bends and bifurcation, where low ESS regions are likely to occur. Indeed, our results demonstrated that as the complexity of the tasks increased, participants were able to maintain their accuracy in the $2 \mathrm{D}$ condition, but not in the $3 \mathrm{D}$ condition.

Additionally, based on the results of this work, our medical participants and collaborators are now convinced of the utility of a 2D data representation and appropriate color map choice:

"Three-dimensional volume visualizations provide the ability to visually follow the connections between different branches. HemoVis presents a surprisingly elegant solution to this problem in 2D by simply and cleanly plotting individual 2D multi-spectral presentations of all vessels concurrently, and simply superposing a graph showing their connectivity. In this manner I think the visualization is a simple yet elegant, and powerful solution for conveying a mix of innately $2 \mathrm{D}$ (stenosis degree) and innately 3D (endothelium) information."

"We have struggled for many years to find a way to display anatomic (i.e., geometric) data and endothelial shear stress data in a comprehensive and intuitive manner. I think HemoVis elegantly solves this problem and should be useful to clinicians and researchers alike. HemoVis is especially helpful in highlighting critical areas of low endothelial shear stress and assessing their relationship to the surrounding anatomy."

"It was surprising to find that different color mapping techniques can render the task of identifying low shear stress regions less ambivalent. By enhancing the perception of identifiable patterns in this complicated problem that spans multiple independent scientific disciplines and hence differently trained scientists, it becomes that much easier to reach significant conclusions. One can only wonder in just how many other instances we make our task more difficult than it needs to be simply by maintaining the status quo. I for one am now more open to consider visualization an integral aspect of research, particularly before dismissing hypotheses that rely on identifying complicated data patterns."

\section{CONCLUSIONS \& FUtURE WORK}

Through our formative qualitative user study, we have developed a task taxonomy for blood flow visualization and we have developed a new 2D tree diagram representation of coronary artery trees. The results of our quantitative study demonstrate that the $2 \mathrm{D}$ representation is not sensitive to increased complexity in the task and users are more accurate and efficient at identifying regions of interest in a 2D representation than a 3D representation, and that the rainbow color map can significantly reduce a person's accuracy and efficiency.

We are continuing to develop HemoVis based on the principles and results of this study. Also, even though the $2 \mathrm{D}$ representation is more accurate and efficient for our tasks, having a 3D representation is still essential for surgical planning. We will investigate the most effective ways to connect these two representations through linked views in future work. We also plan to investigate other user interface designs and interactions for HemoVis. For example, if a doctor were in a clinical setting that allowed for detailed study of the data and interaction, could adding filters to narrow the range of ESS focus or adjustment of color scale parameters be useful.

The work presented in this paper is broadly applicable to other domain applications as well as visualization in general. The new $2 \mathrm{D}$ tree diagram representation utilized in HemoVis is applicable to the visualization of other branched anatomical structures (e.g., cerebral and venous arterial systems, pulmonary systems) and general fluid dynamical pipe structures (e.g., engineering). In terms of general visualization, this work serves as both an example and template of how to convince users of good visualization practices. In this case, a success story of changing users' opinions with particular regard to appropriate dimensionality of data representation and color choice. This work not only shows a real world example demonstrating just how significant an impact rainbow color can have on a user's task, but also a way for other researchers to counter this issue by demonstrating to their users how color impacts their task performance.

\section{ACKNOWLEDGMENTS}

We would like to acknowledge and thank the entire Multiscale Hemodynamics Project team. We would also like to thank Tamara Munzner, Miriah Meyer, and Bernice Rogowitz for their helpful comments on this research and paper. This work was supported in part by Toshiba Medical Systems Corporation (PI FJ Rybicki, MD, PhD), the CyberInfrastructure Lab at the Harvard School of Engineering and Applied Sciences, and NSF Grant PHY-0835713. M. Borkin was supported by the Department of Defense through the National Defense Science \& Engineering Graduate Fellowship (NDSEG) Program, and A. Peters was supported by the Department of Energy through the Computational Science Graduate Fellowship (DOE CSGF) Program.

\section{References}

[1] R. Amar, J. Eagan, and J. Stasko. Low-level components of analytic activity in information visualization. In Proceedings of the Proceedings of the 2005 IEEE Symposium on Information Visualization, pages 15-, Washington, DC, USA, 2005. IEEE Computer Society.

[2] N. Andrienko, G. Andrienko, and P. Gatalsky. Exploratory spatiotemporal visualization: an analytical review. Journal of Visual Languages \& Computing, 14(6):503 - 541, 2003. Visual Data Mining.

[3] B. Bauer, P. Jolicoeur, and W. B. Cowan. Distractor heterogeneity versus linear separability in colour visual search. Perception, 25(11):1281 1293, 1996.

[4] J. Bertin. Semiology of Graphics: Diagrams, Networks, Maps. University of Wisconsin Press, 1983.

[5] D. Borland and R. T. II. Rainbow color map (still) considered harmful. IEEE Computer Graphics and Applications, pages 14-17, 2007.

[6] C. A. Brewer. Color use guidelines for data representation. In Proceedings of the Section on Statistical Graphics, American Statistical Association, pages 55-60, 1999.

[7] K. Bühler, P. Felkel, A. L. Cruz, and R. L. Cruz. Geometric methods for vessel visualization and quantification - a survey. In In Geometric Modelling for Scientific Visualization, pages 399-420. Springer-Verlag, 2002.

[8] Y. S. Chatzizisis, A. U. Coskun, M. Jonas, E. R. Edelman, C. L. Feldman, and P. H. Stone. Role of Endothelial Shear Stress in the Natural History of Coronary Atherosclerosis and Vascular Remodeling: Molecular, Cellular, and Vascular Behavior. J Am Coll Cardiol, 49(25):2379-2393, 2007.

[9] A. Cockburn and B. McKenzie. Evaluating the effectiveness of spatial memory in $2 \mathrm{~d}$ and $3 \mathrm{~d}$ physical and virtual environments. In Proceed- 
ings of the SIGCHI conference, CHI '02, pages 203-210, New York, NY, USA, 2002. ACM.

[10] A. V. Finn, M. Nakano, J. Narula, F. D. Kolodgie, and R. Virmani Concept of vulnerable/unstable plaque. Arterioscler Thromb Vasc Biol, 30(7):1282-1292, 2010.

[11] A. Forsberg, J. Chen, and D. Laidlaw. Comparing 3d vector field visualization methods: A user study. IEEE Transactions on Visualization and Computer Graphics, 15(6):1219-1226, 2009.

[12] A. S. Forsberg, D. H. Laidlaw, A. van Dam, R. M. Kirby, G. E. Karniadakis, and J. L. Elion. Immersive virtual reality for visualizing flow through an artery. In VIS '00: Proceedings of the conference on Visualization '00, pages 457-460, Los Alamitos, CA, USA, 2000. IEEE Computer Society Press.

[13] H. K. Hahn, B. Preim, D. Selle, and H. O. Peitgen. Visualization and interaction techniques for the exploration of vascular structures. In VIS '01: Proceedings of the conference on Visualization '01, pages 395-402, Washington, DC, USA, 2001. IEEE Computer Society.

[14] C. Healey. Choosing effective colours for data visualization. Visualization'96. Proceedings., pages 263-270, 1996.

[15] A. Kanitsar, D. Fleischmann, R. Wegenkittl, P. Felkel, and M. E. Gröller. Cpr: curved planar reformation. In VIS '02: Proceedings of the conference on Visualization '02, pages 37-44, Washington, DC, USA, 2002. IEEE Computer Society.

[16] G. Kindlmann, E. Reinhard, and S. Creem. Face-based luminance matching for perceptual colormap generation. - 2002.

[17] A. Kjellin, L. Pettersson, S. Seipel, and M. Lind. Evaluating 2d and $3 \mathrm{~d}$ visualizations of spatiotemporal information. ACM Transactions on Applied Perception (TAP), 7(3):1-23, 2010.

[18] R. Kosara, C. G. Healey, V. Interrante, D. H. Laidlaw, and C. Ware. User studies: Why, how, and when? IEEE Computer Graphics and Applications, 23:20-25, 2003.

[19] E. L. Koua, A. Maceachren, and M. J. Kraak. Evaluating the usability of visualization methods in an exploratory geovisualization environment. International Journal of Geographical Information Science, 20:425-448(24), April 2006.

[20] A. Kuß, M. Gensel, B. Meyer, V. Dercksen, and S. Prohaska. Effective techniques to visualize filamentsurface relationships. Computer Graphics Forum, 29(3):1003-1012, 2010.

[21] D. Laidlaw, R. Kirby, C. Jackson, J. Davidson, T. Miller, M. D. Silva, W. Warren, and M. Tarr. Comparing $2 \mathrm{~d}$ vector field visualization methods: A user study. IEEE Transactions on Visualization and Computer Graphics, pages 59-70, 2005.

[22] H. Levkowitz and G. T. Herman. Color scales for image data. IEEE Computer Graphics and Applications, 12:72-80, 1992.

[23] A. Light and P. J. Bartlein. The End of the Rainbow? Color Schemes for Improved Data Graphics. EOS Transactions, 85:385-391, Oct. 2004

[24] D. Lloyd-Jones and e. al. Heart Disease and Stroke Statistics-2009 Update: A Report From the American Heart Association Statistics Committee and Stroke Statistics Subcommittee. Circulation, 119(3):e21-181, 2009.

[25] J. Martin, E. S. II, R. J. M. II, Z. Liu, and S. Cai. Results of a user study on 2d hurricane visualization. Computer Graphics Forum, 27(3):991-998, 2008.

[26] S. Melchionna, M. Bernaschi, S. Succi, E. Kaxiras, F. J. Rybicki, D. Mitsouras, A. U. Coskun, and C. L. Feldman. Hydrokinetic approach to large-scale cardiovascular blood flow. Computer Physics Communications, 181(3):462 - 472, 2010.

[27] K. Moreland. Diverging color maps for scientific visualization. Advances in Visual Computing, pages 92-103, 2009.

[28] K. Museth, T. Müller, and A. Ynnerman. Model-free surface visualization of vascular trees. IEEE/Eurographics Symposium on Visualization, 2008.

[29] D. J. Peuquet. It's about time: A conceptual framework for the representation of temporal dynamics in geographic information systems. Annals of the Association of American Geographers, 84(3):441-461, 1994.

[30] C. Plaisant. The challenge of information visualization evaluation. In Proceedings of the working conference on Advanced visual interfaces, AVI '04, pages 109-116, New York, NY, USA, 2004. ACM.

[31] B. Preim and S. Oeltze. 3d visualization of vasculature: An overview, 2008.

[32] P. Rheingans. Color, change, and control for quantitative data display. Proceedings of the 3rd conference on Visualization'92, pages 252-259, 1992.

[33] P. Rheingans. Task-based color scale design (proceedings paper). spie.org, Jan 2000.

[34] F. Ritter, C. Hansen, V. Dicken, O. Konrad, B. Preim, and H. Peitgen. Real-time illustration of vascular structures. IEEE Transactions on Visualization and Computer Graphics, pages 877-884, 2006.

[35] B. Rogowitz and A. Kalvin. The which blair project: A quick visual method for evaluating perceptual color maps. Proceedings of the conference on Visualization'01, pages 183-190, 2001.

[36] B. Rogowitz, A. Kalvin, A. Cohen, and T. Watson. Invited paper: Which trajectories through which perceptually uniform color spaces produce appropriate colors scales for interval data?(gamut mapping i)(report. IS\&T Color Imaging Conference Proceedings, Jan 2000.

[37] B. Rogowitz and L. Treinish. Data visualization: the end of the rainbow. Spectrum, IEEE, 35(12):52-59, 1998.

[38] B. Rogowitz, L. Treinish, and S. Bryson. How not to lie with visualization. Computers in Physics, 10(3):268-273, 1996.

[39] T. Ropinski, S. Hermann, R. Reich, M. Schafers, and K. Hinrichs. Multimodal vessel visualization of mouse aorta pet/ct scans. IEEE Transactions on Visualization and Computer Graphics, 15(6):1515-1522, 2009.

[40] F. J. Rybicki, S. Melchionna, D. Mitsouras, A. U. Coskun, A. G. Whitmore, M. Steigner, L. Nallamshetty, F. G. Welt, M. Bernaschi, M. Borkin, J. Sircar, E. Kaxiras, S. Succi, P. H. Stone, and C. L. Feldman. Prediction of coronary artery plaque progression and potential rupture from 320detector row prospectively ecg-gated single heart beat ct angiography: Lattice boltzmann evaluation of endothelial shear stress. The International Journal of Cardiovascular Imaging, 25(2):289-299, 2009.

[41] J. P. Shaffer. Multiple hypothesis-testing. ANNUAL REVIEW OF PSYCHOLOGY, 46:561-584, 1995

[42] B. Shneiderman. Why not make interfaces better than 3d reality? Computer Graphics and Applications, IEEE, 23(6):12-15, 2003.

[43] P. H. Stone, S. Saito, S. Takahashi, Y. Makita, S. Nakamura, T. Kawasaki, A. Takahashi, T. Katsuki, S. Nakamura, A. Namiki, A. Hirohata, T. Matsumura, S. Yamazaki, H. Yokoi, S. Tanaka, S. Ohtsuji, F. Yoshimachi, J. Honye, D. Harwood, M. Papafaklis, M. Reitman, A. U. Coskun, and C. L. Feldman. The prediction trial: In-vivo assessment of coronary endothelial shear stress, arterial remodeling, and plaque morphology to predict coronary atherosclerosis progression and rupture in man. In Journal of the American College of Cardiology 2011, 2011.

[44] M. Straka, M. Cervenansky, A. La Cruz, A. Kochl, M. Sramek, E. Groller, and D. Fleischmann. The vesselglyph: Focus \& context visualization in ct-angiography. In VIS '04: Proceedings of the conference on Visualization '04, pages 385-392, Washington, DC, USA, 2004. IEEE Computer Society.

[45] M. Termeer, J. O. Bescós, M. Breeuwer, A. Vilanova, F. Gerritsen, and M. E. Gröller. Covicad: Comprehensive visualization of coronary artery disease. IEEE Transactions on Visualization and Computer Graphics (accepted for publication), 13(6):

[46] C. Tominski, G. Fuchs, and H. Schumann. Task-driven color coding. pages 373-380, Jul 2008.

[47] M. Tory, A. Kirkpatrick, and M. Atkins. Visualization task performance with $2 \mathrm{~d}, 3 \mathrm{~d}$, and combination displays. IEEE Transactions on Visualization and Computer Graphics, pages 2-13, 2006.

[48] M. Tory, D. Sprague, F. Wu, W. So, and T. Munzner. Spatialization design: Comparing points and landscapes. IEEE Transactions on Visualization and Computer Graphics, pages 1262-1269, 2007.

[49] C. Ware. Color sequences for univariate maps: Theory, experiments and principles. IEEE Computer Graphics and Applications, pages 41-49, 1988.

[50] C. Ware. Designing with a $21 / 2$-d attitude. Information Design Journal, 10(3):258-65, 2001.

[51] L. Zhu, S. Haker, and A. Tannenbaum. Conformal flattening maps for the visualization of vessels. SPIE Medical Imaging, 4681:742748, 2002.

[52] L. Zhu, S. Haker, and A. Tannenbaum. Flattening maps for the visualization of multibranched vessels. IEEE Transactions on Medical Imaging, 24(2):191-198, 2005 